\title{
Exchange of indivisible goods and indifferences: the Top Trading Absorbing Sets mechanisms*
}

\author{
Jorge Alcalde-Unzu ${ }^{\dagger}$, Elena Molis ${ }^{\ddagger}$
}

December 2010

\begin{abstract}
There is a wide range of economic problems that involve the exchange of indivisible goods with no monetary transfers, starting from the housing market model of the seminal paper by Shapley and Scarf (1974) to problems such as the kidney exchange or the school choice problem. The classical solution to many of these models is to apply a mechanism called Top Trading Cycles, attributed to David Gale, which satisfies good properties for the case of strict preferences. In this paper, we propose a family of mechanisms, called Top Trading Absorbing Sets mechanisms, which generalize the Top Trading Cycles to the general case in which individuals are allowed to report indifferences, while preserving a maximal possible set of its desirable properties.
\end{abstract}

JEL classification: $\mathrm{C} 71 ; \mathrm{C} 78 ; \mathrm{D} 71 ; \mathrm{D} 78$

Keywords: housing market; indifferences; top trading cycles; absorbing sets

\footnotetext{
${ }^{*}$ We are very grateful to Miguel A. Ballester, Salvador Barberá, Peter Biró, Aytek Erdil, Elena Inarra, Onur Kesten, Jordi Massó, Clara Ponsati, Jay Sethuraman, Federico Valenciano, Allard van der Made, Mathieu van Vyve, Vincent Vannetelbosch, the audience of the seminars in Bilbao and Málaga, the participants at the IAP Workshop in Leuven and at the matching workshop in Oxford for their very helpful comments and suggestions. We are particularly grateful to two anonymous referees and the Advisory Editor for many valuable comments and suggestions that have led to a substantial improvement in the manuscript. Authors acknowledge financial support from the Spanish Ministry of Education and Science, through the projects ECO2009-11213 and ECO2009-12836, from Basque Government through the project GIC07/146-IT-377-07, from Andalusian Government through the project P07.SEJ.02547 and from Belgian Federal Government through the IAP Project (contract 6/09).

${ }^{\dagger}$ Public University of Navarre, Department of Economics. Campus Arrosadia, 31006 Pamplona, Spain. E-mail: jorge.alcalde@unavarra.es.

${ }^{\ddagger}$ Universidad de Granada, Department of Economics, Campus la Cartuja, 18011 Granada, Spain. emolis@ugr.es
} 


\section{Introduction}

Shapley and Scarf (1974) model a simple economy in which there is a set of agents, each of whom has strict preferences over a set of indivisible goods, such as houses. In such an economy, commonly known as a "housing market", agents are endowed with one house each and are allowed to swap houses among themselves, although monetary transfers are not permitted. In their seminal paper, Shapley and Scarf prove that this economy has always a non-empty strict core by using a so-called Top Trading Cycles Algorithm (hereafter, TTC), attributed to David Gale.

The housing market has been extensively analyzed in the literature on the domain of strict preferences. ${ }^{1}$ It has become plain that the TTC mechanism satisfies very desirable properties. Roth and Postlewaite (1977) prove that this mechanism results in the unique assignment belonging to the strict core and, additionally, that this assignment is the unique competitive allocation. Subsequently, Roth (1982) shows that it is a dominant strategy for agents to reveal their true preferences in this mechanism. Bird (1984) shows that this mechanism is also invulnerable to group manipulations of the preferences. Furthermore, Ma (1994) shows that the TTC mechanism (also known as the strict core mechanism) is the only mechanism satisfying individual rationality, Pareto-efficiency and strategy-proofness on this domain.

In contrast, very few papers have been written on the housing market on the full preference domain, even though it seems quite natural for agents to be indifferent between goods. One possible reason for this is that the consideration of weak preferences in the model introduces additional complications. First of all, in this case, the strict core might be empty, unique or multi-valued. Moreover, although the core is always non-empty, some of its allocations might be inefficient. Additionally, some of the properties that are satisfied by the TTC mechanism on the restricted domain of strict preferences are incompatible on the full preference domain. For instance, Ehlers (2002) shows that Pareto-efficiency and group strategy-proofness are not compatible. We will additionally show that on this general domain there may be housing market problems without Pareto-efficient competitive allocations.

As far as we know, there are two preceding papers proposing algorithms and mechanisms to solve the housing market problem on the full preference domain. ${ }^{2}$ The first, by Quint and Wako (2004), proposes an algorithm to determine whether or not the strict core is empty and obtain a strict core assignment if it is non-empty. Nevertheless, this cannot be considered a mechanism, since, for housing markets with an empty strict core, it reports that the strict core is empty but it does not give any allocation. The other, by Yllmaz (2009), presents a random mechanism satisfying individual rationality, ex-ante efficiency and no justified-envy. However, this is not a strict core mechanism (i.e., there are housing market problems with a non-empty strict core in which the mechanism does not select a strict core allocation). This mechanism is not a generalization of the TTC mechanism either, since the allocation it proposes for problems with strict preferences may be different from the unique strict core allocation. Furthermore, this mechanism does not satisfy strategy-proofness, although it attains higher levels of efficiency.

\footnotetext{
${ }^{1}$ For a comprehensive survey on housing market with strict preferences see Sönmez and Ünver (2009).

${ }^{2}$ Konishi et al. (2001) study the case in which goods are of different types and all individuals are indifferent only between goods of the same type. This implies that individuals are not allowed to have different indifference classes. Consequently, they work on a domain that is richer than the classical strict preference domain, but more restrictive than the full preference domain.
} 
The contribution of this paper is to present a family of mechanisms that generalize the TTC mechanism while preserving some of their good properties when agents are allowed to report indifferences. In order to introduce this family of mechanisms, we define an algorithm called Top Trading Absorbing Sets algorithm (hereafter TTAS), which results in a strict core allocation when the strict core is non-empty and a Pareto-efficient core allocation otherwise. Then, we prove that the family of mechanisms constructed using this algorithm satisfy individual rationality, Pareto-efficiency and strategy-proofness. Given that the other classical properties satisfied by the TTC on the domain of strict preferences (group strategy-proofness and the selection of competitive allocations) are incompatible on this general domain with efficiency, what we are presenting in this paper is a generalization of the TTC mechanism maintaining a maximal possible set of its properties for the case of strict preferences. ${ }^{3}$

The literature also describes other problems where there are indivisible goods and monetary transfers are not allowed. Some examples are housing allocation with existing tenants (Abdulkadiroğlu and Sönmez (1999)), kidney exchange (Roth et al. (2004)) and school choice (Abdulkadiroğlu and Sönmez (2003)). In the cited problems, the solution, or one of the proposed solutions for such problems, is based on an adaptation of the TTC to the corresponding framework. However, as in the housing market problem, they only address the case in which agents have strict preferences. Our family of mechanisms (with the same particular adaptations needed for each framework) will generalize all these adaptations of the TTC mechanism for these particular problems to the case in which agents are allowed to report indifferences.

The rest of the paper is organized as follows. Section 2 contains some basic preliminaries of the housing market problem and graph theory. Section 3 revises the TTC mechanism, introduces the family of TTAS mechanisms and studies the properties of this family of mechanisms in the housing market problem. Section 4 discusses some characteristics of the mechanisms of the family and presents some further applications of them. Finally, the proofs of the results obtained throughout the paper are given in the appendix.

\section{The housing market model}

Let $N$ be a finite set of agents and $H$ be a finite set of houses such that $|N|=|H|=n$. Each individual $i \in N$ has a transitive and complete (but not necessarily antisymmetric) preference binary relation $R_{i}$ over $H$. As usual, $P_{i}$ and $I_{i}$ will be used to denote the asymmetric and symmetric parts of $R_{i}$, respectively. Let $R=\left(R_{i}\right)_{i \in N}$ be the preference profile. Given $S \subset N$, let $R_{-S}=\left(R_{j}\right)_{j \in N \backslash S}$ denote the preferences of all individuals except those in $S$. By abusing notation, we will use $R_{-i}$ instead of $R_{-\{i\}}$. For any $R_{i}$ and any $S \subseteq H$, we will define the maximal elements of $S$ according to $R_{i}$ as the set $\max _{S}\left(R_{i}\right)=\{x \in$ $S \mid x R_{i} y$ for all $\left.y \in S\right\}$.

An assignment (or allocation) is a bijective map $\mu: N \longrightarrow H$. Let $\mu(i)$ denote the house assigned to agent $i$ under the assignment $\mu$. By abusing notation, we will also use $\mu_{i}$ instead of $\mu(i)$. The assignment describing the initial owners of houses is called the "initial endowment" and is denoted by $\omega$. For any $T \subseteq N$, we define $\omega(T)=\left\{x \in H \mid x=\omega_{i}\right.$ for some $\left.i \in T\right\}$. Then, a housing market is a list $(N, H, \omega, R)$.

\footnotetext{
${ }^{3}$ Jaramillo and Manjunath (2009) have a simultaneous and independent work to this one presenting another family of mechanisms generalizing the TTC mechanism with similar properties.
} 
A deterministic mechanism $f$ is a map that assigns for each housing market $(N, H, \omega, R)$ an assignment $f(N, H, \omega, R)$. When the description of $(N, H, \omega, R)$ is clear, we will denote the house assigned by the mechanism $f$ to agent $i$ by $f_{i}$. Let $\mathcal{F}$ be the set of all deterministic mechanisms. A random mechanism $g$ is a probability distribution over $\mathcal{F}$. That is, a random mechanism associates to each housing market a probability distribution over the set of assignments. Note that any deterministic mechanism is a random mechanism.

An assignment $\mu$ is individually rational if, for each agent $i \in N, \mu_{i} R_{i} \omega_{i}$. A deterministic mechanism $f$ is individually rational if it always selects an individually rational assignment for each housing market. A random mechanism is individually rational if its support contains only individually rational deterministic mechanisms.

An assignment $\mu$ is Pareto-efficient if no other assignment $\nu$ exists such that, for all $i \in N, \nu_{i} R_{i} \mu_{i}$ and for some $j \in N, \nu_{j} P_{j} \mu_{j}$. A deterministic mechanism $f$ is Pareto-efficient if it always selects a Pareto-efficient assignment for each housing market. A random mechanism is ex-post efficient if its support contains only Pareto-efficient deterministic mechanisms. A random mechanism $g$ stochastically dominates another random mechanism $h$ if, for any possible vector of utilities $U=\left(u_{i}\right)_{i \in N}$ compatible with $R$, the following must hold: for all $i \in N$,

$\sum_{x \in H} p\left(g_{i}(N, H, \omega, R)=x\right) \cdot u_{i}(x) \geq \sum_{x \in H} p\left(h_{i}(N, H, \omega, R)=x\right) \cdot u_{i}(x)$ and there is some $j \in N$ for which this inequality is strict.

Then, a random mechanism $g$ is ex-ante efficient if it is not stochastically dominated by any other random mechanism.

A random mechanism $g$ is strategy-proof if truth-telling is a dominant strategy in its associated preference revelation game. That is, for all $R$ and for any possible vector of utility functions $U=\left(u_{i}\right)_{i \in N}$ compatible with $R$, the following must hold: for all $i \in N$,

$$
\sum_{x \in H} p\left(g_{i}(N, H, \omega, R)=x\right) \cdot u_{i}(x) \geq \sum_{x \in H} p\left(g_{i}\left(N, H, \omega,\left(R_{-i}, R_{i}^{\prime}\right)\right)=x\right) \cdot u_{i}(x) \text { for all possible } R_{i}^{\prime} .
$$

A random mechanism $g$ is group strategy-proof if for all $R$ and for any possible vector of utility functions $U=\left(u_{i}\right)_{i \in N}$ compatible with $R$, the following must hold: for all $S \subset N$, there exists $i \in S$ such that

$$
\sum_{x \in H} p\left(g_{i}(N, H, \omega, R)=x\right) \cdot u_{i}(x) \geq \sum_{x \in H} p\left(g_{i}\left(N, H, \omega,\left(R_{-S}, R_{S}^{\prime}\right)\right)=x\right) \cdot u_{i}(x) \text { for all possible } R_{S}^{\prime} .
$$

An assignment $\mu$ is competitive if a price vector $p=\left(p_{1}, \ldots, p_{n}\right)$ exists such that for all $i \in N, p_{\mu_{i}} \leq$ $p_{\omega_{i}}$ (budget constraint) and $\mu_{i} R_{i} \omega_{j}$ for all $j \in N$ with $p_{\omega_{j}} \leq p_{\mu_{i}}$ (utility maximization). Intuitively, an assignment is competitive if a set of prices exists such that the assignment can be explained by a competitive equilibrium. A deterministic mechanism $f$ is competitive if it always selects a competitive assignment for each housing market. A random mechanism is competitive if its support contains only competitive deterministic mechanisms.

An assignment $\mu$ is in the core of the housing market if there is no coalition $T \subseteq N$ and assignment $\nu$ such that, for all $i \in T, \nu_{i} \in \omega(T)$ and $\nu_{i} P_{i} \mu_{i}$. An assignment $\mu$ is in the strict core of the housing market if there is no coalition $T \subseteq N$ and assignment $\nu$ such that, for all $i \in T, \nu_{i} \in \omega(T)$ and $\nu_{i} R_{i} \mu_{i}$ and for some $j \in T, \nu_{j} P_{j} \mu_{j}$. 


\section{Preliminaries in digraphs}

A directed graph, or digraph, is a pair $(V, E)$, where $V$ is a non-empty set of nodes and $E$ is a set of directed arcs (or edges). The indegree (outdegree) of a node $v_{i} \in V$ is the number of arcs leading to (leading from) $v_{i}$. Given two nodes $v_{i}, v_{j} \in V$, we say that there is a path from $v_{i}$ to $v_{j}$ if there is a sequence of nodes $v_{i}=v_{1}, \ldots, v_{m}=v_{j}$ such that for all $i \in\{1, \ldots, m-1\}$, there is an arc from $v_{i}$ to $v_{i+1}$. A chain is an ordered set of nodes $C=\left\{v_{1}, v_{2}, \ldots, v_{m}\right\}$ such that for all $i \in\{1, \ldots, m-1\}$, there is an arc from $v_{i}$ to $v_{i+1}$ and there is not an arc from $v_{m}$ to $v_{1}$. A cycle is an ordered set of nodes $C=\left\{v_{1}, v_{2}, \ldots, v_{m}\right\}$ such that for all $i \in\{1, \ldots, m-1\}$, there is an arc from $v_{i}$ to $v_{i+1}$ and there is an arc from $v_{m}$ to $v_{1}$. Two nodes $v_{i}, v_{j} \in V$ constitute a symmetric pair if there is an arc from $v_{i}$ to $v_{j}$ and an arc from $v_{j}$ to $v_{i}$. Given two digraphs $(V, E),\left(V^{\prime}, E^{\prime}\right)$, we say that $\left(V^{\prime}, E^{\prime}\right)$ is a subgraph of $(V, E)$ if $V^{\prime} \subseteq V$ and $E^{\prime} \subseteq E$.

An absorbing set is a set of nodes $A$ that satisfies two conditions: (i) for any two nodes $v_{i}, v_{j} \in A$, there is a path from one to the other (inside connection), and (ii) there is no path from any node $v_{i} \in A$ to any node $v_{j} \notin A$ (no inside-outside connection). An absorbing set is paired-symmetric if each of its nodes belongs to a symmetric pair.

\section{Mechanisms}

The classical framework in which the housing market problem is studied in the literature consists of individuals having strict preferences. Shapley and Scarf (1974) show that, in this case, the strict core always exists and they propose the Strict Core mechanism, which selects the unique strict core assignment for each housing market. ${ }^{4}$ This deterministic mechanism is strategy-proof, as Roth (1982) show. Moreover, Ma (1994) shows that this is the only mechanism that satisfies individual rationality, Pareto-efficiency and strategy-proofness on the domain of strict preferences. Shapley and Scarf (1974) attribute to David Gale the algorithm called Top Trading Cycles to compute the strict core assignment of a housing market.

\section{The Top Trading Cycles mechanism}

Consider a directed graph in which there are two types of nodes (agents and houses) and arcs leading from agents to houses and from houses to agents, and all nodes have outdegree equal to 1 . An interesting fact about any directed graph with these characteristics is that it always has at least one cycle and no two cycles intersect. This implies that the following algorithm, called Top Trading Cycles, always determines an assignment.

Gale's Top Trading Cycles (TTC) algorithm:

\section{Step 1:}

(1.1) Let each agent point to her maximal house and each house point to its owner. Select the cycles of this graph.

(1.2) The agents in the cycles are removed from the algorithm by assigning to each of them the house she is pointing to.

\footnotetext{
${ }^{4}$ Roth and Postlewaite (1977) prove that the strict core assignment is unique for housing markets with strict preferences.
} 


\section{Step $i$ :}

(i.1) Let each remaining agent point to her maximal house from among the remaining ones and each remaining house point to its owner (note that when an agent leaves, her original house also leaves, so if a house remains in the algorithm then its owner also remains and vice versa). Select the cycles of this graph.

(i.2) The agents in the cycles are removed from the algorithm by assigning to each of them the house she is pointing to.

The algorithm terminates when no agents and houses remain, and the outcome is the assignment formed during its execution.

In the general case, in which indifferences are allowed, the strict core may not be unique. Indeed, it may be multi-valued and even empty. Quint and Wako (2004) propose an algorithm, called Top Trading Segmentation which determines whether a housing market problem has an empty strict core or not and, in the event of having a non-empty strict core, it finds an allocation belonging to it. Given that all the allocations of the strict core are indifferent for all individuals (i.e., if $\mu$ and $\rho$ belong to the strict core, $\mu_{i} I_{i} \rho_{i}$ for all $\left.i \in N\right)$, this algorithm provides a good solution for the case in which the strict core is nonempty. However, no satisfactory mechanism extending the TTC exists that works for every housing market problem, independently of having a non-empty strict core or not. ${ }^{5}$ A mechanism suggested in the literature to generalize the TTC mechanism (see, for example, Roth (1982)) is the following: (1) Take the preferences of those agents having indifferences and turn them into strict orders by means of (fixed or random) tie-breakers; and (2) apply the Top Trading Cycles mechanism.

It is clear that this class of mechanisms coincide with the TTC for the case of strict preferences. However, in the case of allowing agents to report indifferences, the application of these mechanisms does not necessarily lead to efficient assignments. There are, in fact, cases where efficient assignments are never achieved, independently of the choice of tie-breakers. We illustrate this with the following example.

Example 1 Let $N=\left\{a_{1}, a_{2}, a_{3}, a_{4}, a_{5}\right\}$ and $H=\left\{h_{1}, h_{2}, h_{3}, h_{4}, h_{5}\right\}$ be the set of agents and houses, respectively. Let $\omega\left(a_{i}\right)=h_{i}$ for all $i \in\{1, \ldots, 5\}$ be the initial endowment. Let the preference profile be as follows:

\begin{tabular}{ccccc}
$a_{1}$ & $a_{2}$ & $a_{3}$ & $a_{4}$ & $a_{5}$ \\
\hline$h_{2}$ & $h_{3}$ & $h_{4}, h_{5}$ & $h_{1}$ & $h_{2}$ \\
$h_{1}$ & $h_{2}$ & $h_{3}$ & $h_{5}$ & $h_{4}$ \\
$h_{3}$ & $h_{1}$ & $h_{1}$ & $h_{4}$ & $h_{5}$ \\
$h_{4}$ & $h_{4}$ & $h_{2}$ & $h_{2}$ & $h_{1}$ \\
$h_{5}$ & $h_{5}$ & & $h_{3}$ & $h_{3}$
\end{tabular}

In this housing market problem, the strict core is empty and the core contains the following four allocations:

\footnotetext{
${ }^{5}$ Yılmaz (2009) proposes a mechanism, but it is not a generalization of the TTC mechanism.
} 


$$
\begin{aligned}
& \mu^{1}=\left\{\left(a_{1}, h_{2}\right),\left(a_{2}, h_{3}\right),\left(a_{3}, h_{4}\right),\left(a_{4}, h_{1}\right),\left(a_{5}, h_{5}\right)\right\} \\
& \mu^{2}=\left\{\left(a_{1}, h_{1}\right),\left(a_{2}, h_{3}\right),\left(a_{3}, h_{5}\right),\left(a_{4}, h_{4}\right),\left(a_{5}, h_{2}\right)\right\} \\
& \mu^{3}=\left\{\left(a_{1}, h_{2}\right),\left(a_{2}, h_{3}\right),\left(a_{3}, h_{5}\right),\left(a_{4}, h_{1}\right),\left(a_{5}, h_{4}\right)\right\} \\
& \mu^{4}=\left\{\left(a_{1}, h_{1}\right),\left(a_{2}, h_{3}\right),\left(a_{3}, h_{4}\right),\left(a_{4}, h_{5}\right),\left(a_{5}, h_{2}\right)\right\}
\end{aligned}
$$

There is only one indifference binary relation in the preference profile and, then, there are two possible results of the class of mechanisms presented above, namely $\mu^{1}$ and $\mu^{2}$. However, it is easy to see that these allocations are Pareto dominated by $\mu^{3}$ and $\mu^{4}$, respectively.

We will next show that none of the Pareto-efficient assignment of the housing market problem in Example $1, \mu^{3}$ and $\mu^{4}$, are competitive assignments. Notice that for $\mu^{3}$ being a competitive assignment, it is necessary that $p_{\mu^{3}\left(a_{1}\right)}=p_{h_{2}} \leq p_{h_{1}}$ and $p_{\mu^{3}\left(a_{4}\right)}=p_{h_{1}} \leq p_{h_{4}}$ and, therefore, $p_{h_{2}} \leq p_{h_{4}}$. However, since $h_{2} P_{5} h_{4}$ (where

$h_{4}=\mu^{3}\left(a_{5}\right)$ ), then $p_{h_{2}}>p_{h_{4}}$, which is not possible. (The analysis to prove that $\mu_{4}$ is not a competitive equilibrium is similar). Hence, the previous example is also useful to prove the following result:

Remark 1 There is no random mechanism that is competitive and satisfies ex-post efficiency.

We also know from Ehlers (2002) that there is no mechanism that is ex-post efficient and group strategyproof on the general domain of preferences.

Then, we have that, (i) the TTC mechanism performs well for the restricted domain of strict preferences, but the application of tie-breakers is not an efficient solution for the general case; (ii) the Top Trading Segmentation algorithm provides a solution for some cases on the general domain, but it is not a mechanism in the sense that it provides no allocation when the strict core is empty; and (iii) efficiency is incompatible with other properties such as group strategy-proofness and competitiveness. Our objective is to propose a family of mechanisms, called Top Trading Absorbing Sets, for the general domain, that extend the TTC and the TTS satisfying a maximal possible set of the properties that the TTC has. More concretely, in the dichotomies of (iii), our family of mechanisms satisfy ex-post efficiency, and renounce the properties of group strategy-proofness and competitiveness.

\section{Top Trading Absorbing Sets mechanisms}

To introduce the algorithm that determines the family of mechanisms presented in this paper, we use a similar approach to the TTC algorithm. Consider a directed graph in which there are two types of nodes (agents and houses) with arcs leading from agents to houses and from houses to agents, all nodes having strictly positive outdegree. An interesting characteristic of these digraphs is that they always have at least one absorbing set (see Kalai and Schmeidler (1977)).

The Top Trading Absorbing Sets (TTAS) algorithm:

Step 0: Consider a priority ranking of the houses; i.e., a complete, transitive and antisymmetric binary relation over $H$.

\section{Step 1:}

(1.1) Let each agent point to her maximal houses and each house point to its owner. Select the absorbing sets of this graph. 
(1.2) Consider the paired-symmetric absorbing sets, if any. Their agents are removed from the algorithm by assigning to each of them her initial endowment (Obviously, their houses are also removed).

(1.3) Consider the remaining absorbing sets, if any. For each agent pointing to more than one house, select a unique house to point to using the following criterion: she points to the maximal house with the highest priority, different from her initial endowment.

(1.4) Then, in the subgraph formed by the absorbing sets and the arcs selected in step (1.3), there is necessarily at least one cycle and no two cycles intersect. Assign provisionally to each agent in these cycles the house she is pointing to, but keep them in the algorithm.

\section{Step $i$ :}

(i.1) Let each remaining agent point to her maximal houses from among the remaining ones and each house point to its current owner. Select the absorbing sets of this graph.

(i.2) Consider the paired-symmetric absorbing sets, if any. Their agents are removed from the algorithm by assigning to each of them her current endowment (Obviously, their houses are also removed).

(i.3) Consider the remaining absorbing sets, if any. For each agent pointing to more than one house, select a unique house to point to using the following criterion: she points to the maximal house with the highest priority from among those that have not been assigned to her yet. If all maximal houses has been assigned to her at least $m$ times, then she points to the maximal house with the highest priority that has not been assigned to her $\mathrm{m}+1$ times yet. ${ }^{6}$

(i.4) Then, in the subgraph formed by the absorbing sets and the arcs selected in step (i.3), there is necessarily at least one cycle and no two cycles intersect. Assign provisionally to each agent in these cycles the house she is pointing to, but keep them in the algorithm.

The algorithm terminates when no agents and houses remain, and the outcome is the assignment formed during its execution.

The following example illustrates how the TTAS algorithm works for a particular housing market problem.

Example 2 Consider a housing market with $N=\left\{a_{1}, a_{2}, \ldots, a_{10}\right\}$ and $H=\left\{h_{1}, h_{2}, \ldots, h_{10}\right\}$ and assume that the initial endowment of agent $a_{i}$ is house $h_{i}$ for all $i \in\{1,2, \ldots, 10\}$. Let the preference profile $R$ be as follows (we only include, for each agent, the houses that are not worse than her initial endowment):

\begin{tabular}{cccccccccc}
$a_{1}$ & $a_{2}$ & $a_{3}$ & $a_{4}$ & $a_{5}$ & $a_{6}$ & $a_{7}$ & $a_{8}$ & $a_{9}$ & $a_{10}$ \\
\hline$h_{2}$ & $h_{3}$ & $h_{4}, h_{5}$ & $h_{1}$ & $h_{6}$ & $h_{6}, h_{7}$ & $h_{6}$ & $h_{5}, h_{9}$ & $h_{9}, h_{10}$ & $h_{9}, h_{10}$ \\
$h_{1}$ & $h_{2}$ & $h_{3}$ & $h_{5}$ & $h_{2}$ & & $h_{7}$ & $h_{8}$ & & \\
& & & $h_{4}$ & $h_{4}$ & & & & & \\
& & & & $h_{5}$ & & & & &
\end{tabular}

Consider the following priority ranking of houses:

$$
h_{1} \succ h_{2} \succ h_{3} \succ h_{4} \succ h_{5} \succ h_{6} \succ h_{7} \succ h_{8} \succ h_{9} \succ h_{10} .
$$

\footnotetext{
${ }^{6}$ We understand that the initial endowment of an agent is always one of her previously assigned houses.
} 
We depict the directed graphs that are formed at each step of the algorithm: 7

\section{Step 1:}

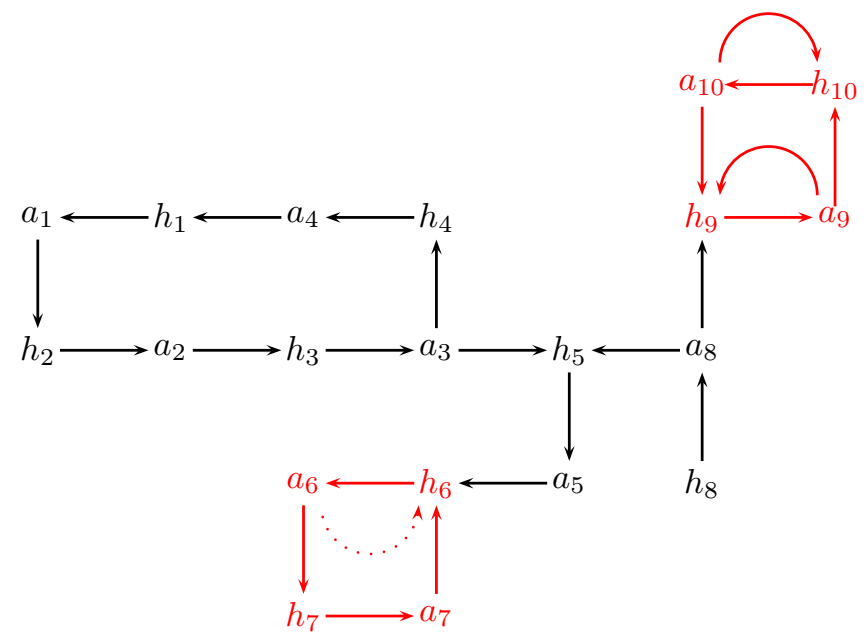

There are two absorbing sets: $A_{1}^{*}=\left\{a_{9}, h_{9}, a_{10}, h_{10}\right\}$, which is paired-symmetric and, hence, this is removed by assigning $h_{9}$ to $a_{9}$ and $h_{10}$ to $a_{10}$. The other absorbing set is $A_{2}=\left\{a_{7}, h_{7}, a_{6}\right.$, $\left.h_{6}\right\}$. In this case, the priority ranking over houses is applied, and the cycle $c=\left(a_{6}, h_{7}, a_{7}, h_{6}\right)$ is formed. Then, the algorithm provisionally assigns $h_{7}$ to $a_{6}$ and $h_{6}$ to $a_{7}$.

\section{Step 2:}

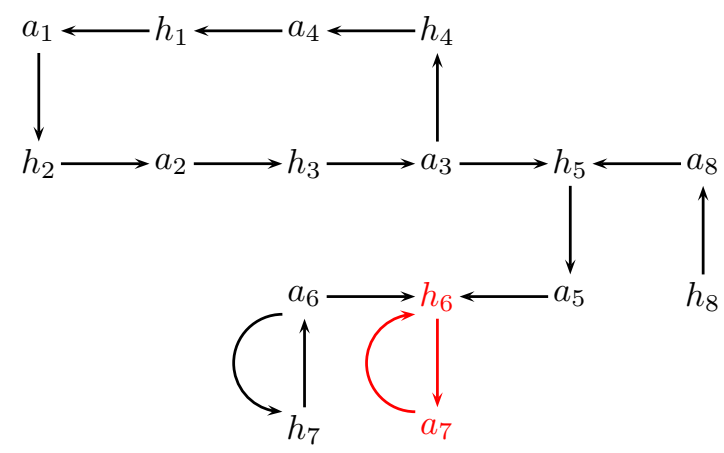

There is only one absorbing set: $A_{3}^{*}=\left\{h_{6}, a_{7}\right\}$, which is paired-symmetric. This is removed by assigning $h_{6}$ to $a_{7}$.

\footnotetext{
${ }^{7}$ There are two colors for the arcs in each digraph: black arcs are those which do not join nodes in an absorbing set; and red arcs are those which do. Within the set of red arcs, there are two types: those drawn with a dotted line are the arrows that are not selected by the priority criterion in step $(i .3)$; and those drawn with a continuous line are the ones chosen by the priority criterion.
} 
Step 3:

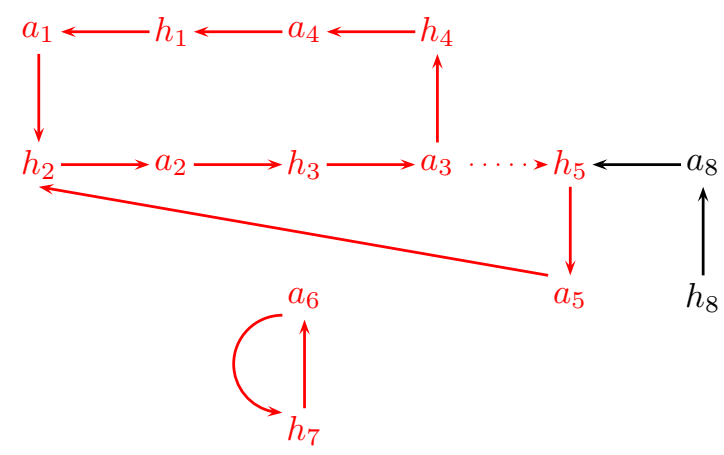

There is a paired-symmetric absorbing set $A_{4}^{*}=\left\{a_{6}, h_{7}\right\}$, which is removed by assigning $h_{7}$ to $a_{6}$. There is another absorbing set $A_{5}=\left\{a_{1}, h_{1}, a_{2}, h_{2}, a_{3}, h_{3}, a_{4}, h_{4}, a_{5}, h_{5}\right\}$. By applying the priority ranking, the cycle $c=\left(a_{1}, h_{2}, a_{2}, h_{3}, a_{3}, h_{4}, a_{4}, h_{1}\right)$ is formed. Then, the algorithm provisionally assigns $h_{2}$ to $a_{1}, h_{3}$ to $a_{2}, h_{4}$ to $a_{3}$ and $h_{1}$ to $a_{4}$.

\section{Step 4:}

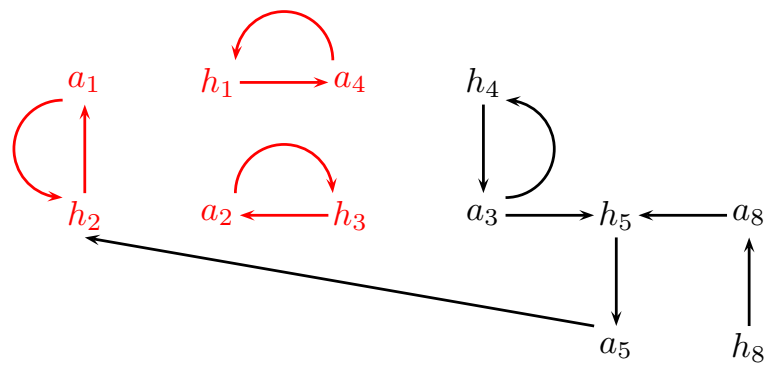

There are 3 paired-symmetric absorbing sets $A_{6}^{*}=\left\{a_{1}, h_{2}\right\}, A_{7}^{*}=\left\{a_{2}, h_{3}\right\}$ and $A_{8}^{*}=\left\{a_{4}, h_{1}\right\}$, which are removed by assigning $h_{2}$ to $a_{1}, h_{3}$ to $a_{2}$ and $h_{1}$ to $a_{4}$, respectively.

\section{Step 5:}

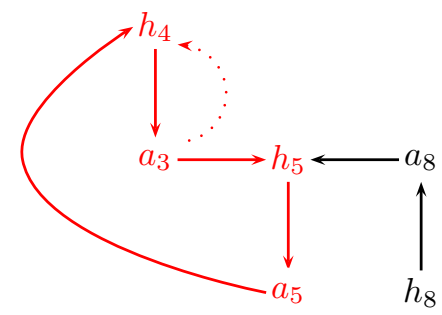


There is only one absorbing set $A_{9}=\left\{a_{3}, h_{4}, a_{5}, h_{5}\right\}$, which is not paired-symmetric. In this case, the cycle $c=\left(a_{3}, h_{5}, a_{5}, h_{4}\right)$ is obtained by applying the criterion of step (5.3). Then, the algorithm provisionally assigns $h_{5}$ to $a_{3}$ and $h_{4}$ to $a_{5}$, respectively.

\section{Step 6:}

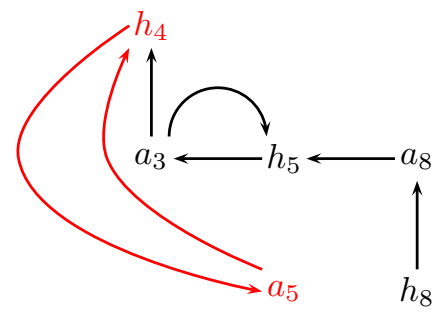

There is a paired-symmetric absorbing set $A_{10}^{*}=\left\{a_{5}, h_{4}\right\}$, which is removed by assigning $h_{4}$ to $a_{5}$.

\section{Step 7:}

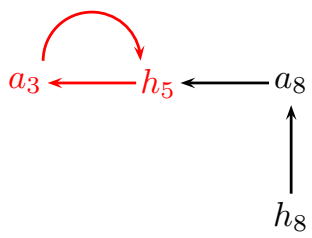

There is a paired-symmetric absorbing set $A_{11}^{*}=\left\{a_{3}, h_{5}\right\}$, which is removed by assigning $h_{5}$ to $a_{3}$.

\section{Step 8:}

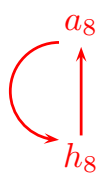

There is a paired-symmetric absorbing set $A_{11}^{*}=\left\{a_{8}, h_{8}\right\}$, which is removed by assigning $h_{8}$ to $a_{8}$. Therefore, the allocation determined by the algorithm is

$$
\mu=\left\{\left(a_{1}, h_{2}\right),\left(a_{2}, h_{3}\right),\left(a_{3}, h_{5}\right),\left(a_{4}, h_{1}\right),\left(a_{5}, h_{4}\right),\left(a_{6}, h_{7}\right),\left(a_{7}, h_{6}\right),\left(a_{8}, h_{8}\right),\left(a_{9}, h_{9}\right),\left(a_{10}, h_{10}\right)\right\} .
$$


The following proposition shows that the TTAS always selects an assignment.

Proposition 1 The TTAS algorithm always provides an allocation.

It is easy to see that in the case in which all individuals have strict preferences, all absorbing sets that appear at any step $i$ of the algorithm are cycles and that, when the trading between agents in each cycle is carried out, each agent forms a paired-symmetric absorbing set with her new house at step $i+1$ and leaves the algorithm. As a consequence, the assignment of the TTAS coincides with the one of the TTC when the preferences are strict.

The TTAS algorithm determines an allocation depending on the priority ranking $\succ$ selected at Step 0 . Then, we define a mechanism for each priority ranking as follows: a mechanism $f$ is a Top Trading Absorbing Sets mechanism if a priority ranking $\succ$ exists such that the mechanism selects, for each housing market problem, the assignment selected by the TTAS algorithm with this priority ranking. We will denote this mechanism by $\sigma^{\succ}$.

In what follows, we will prove some properties of our family of mechanisms. First, we show that it always selects an assignment in the core of the housing market.

Theorem 1 Let $(N, H, \omega, R)$ be a housing market. For any priority ranking $\succ$, the mechanism $\sigma^{\succ}$ selects an assignment in the core of $(N, H, \omega, R)$.

As a corollary, we can deduce that all TTAS mechanisms satisfy individual rationality.

Corollary 1 For any priority ranking $\succ$, the mechanism $\sigma^{\succ}$ is individually rational.

Now, we prove that the TTAS mechanism maintains, on the general domain, some of the properties that characterize the TTC mechanism on the restricted domain of strict preferences. We start with Paretoefficiency.

Theorem 2 For any priority ranking $\succ$, the mechanism $\sigma^{\succ}$ is Pareto-efficient.

Additionally, we also prove that any mechanism belonging to our family is strategy-proof.

Theorem 3 For any priority ranking $\succ$, the mechanism $\sigma^{\succ}$ is strategy-proof.

Then, we have proved that, while in the restricted case of strict preferences the TTC mechanism is the only one satisfying individual rationality, Pareto-efficiency and strategy-proofness (see Ma (1994)), in the general case, we have at least this family of mechanisms satisfying all these properties. Additionally, we will prove that our family of mechanisms always select a strict core assignment if the strict core is non-empty. That is, our family of mechanisms generalize the solution of Quint and Wako (2004).

Theorem 4 Let $(N, H, \omega, R)$ be a housing market with a non-empty strict core. Then, for any priority ranking $\succ$, the mechanism $\sigma^{\succ}$ selects a strict core assignment. 


\section{Comments and applications}

\subsection{Impact and selection of the priority ranking}

We have proposed a family of mechanisms for housing market problems in the general case in which individuals are allowed to report indifferences. This family of mechanisms generalize the previous TTC mechanism and the TTS algorithm, maintaining all the possible desirable properties without renouncing Pareto-efficiency satisfied by these proposals. The mechanisms of this family differ among themselves in the priority ranking, implemented to favor some individuals over others in the event of conflict. The impact of the chosen priority ranking on the assignment provided by a TTAS mechanism varies depending on the housing market problem under consideration. If the problem has a non-empty strict core, all agents are indifferent between the different assignments proposed by the mechanisms of the family, independently of the selected priority ranking. However, when the problem has an empty strict core, the assignments proposed by the different mechanisms can be very different for some agents, as the following example shows:

Example 3 Let $N=\left\{a_{1}, \ldots, a_{8}\right\}$ and $H=\left\{h_{1}, \ldots, h_{8}\right\}$ be the set of agents and houses. Let $\omega\left(a_{i}\right)=h_{i}$ for all $i \in\{1, \ldots, 8\}$ be the initial endowment. The preference profile is the following (we only include, for each agent, the houses that are not worse than her initial endowment):

\begin{tabular}{cccccccc}
$a_{1}$ & $a_{2}$ & $a_{3}$ & $a_{4}$ & $a_{5}$ & $a_{6}$ & $a_{7}$ & $a_{8}$ \\
\hline$h_{2}$ & $h_{3}$ & $h_{1}, h_{4}$ & $h_{6}$ & $h_{3}$ & $h_{5}, h_{7}$ & $h_{8}$ & $h_{6}$ \\
$h_{1}$ & $h_{2}$ & $h_{3}$ & $h_{4}$ & $h_{5}$ & $h_{6}$ & $h_{7}$ & $h_{8}$
\end{tabular}

In this problem, the strict core is empty and the core contains the following two Pareto-efficient allocations:

$$
\begin{aligned}
& \mu^{1}=\left\{\left(a_{1}, h_{2}\right),\left(a_{2}, h_{3}\right),\left(a_{3}, h_{1}\right),\left(a_{4}, h_{4}\right),\left(a_{5}, h_{5}\right),\left(a_{6}, h_{7}\right),\left(a_{7}, h_{8}\right),\left(a_{8}, h_{6}\right)\right\} \\
& \mu^{2}=\left\{\left(a_{1}, h_{1}\right),\left(a_{2}, h_{2}\right),\left(a_{3}, h_{4}\right),\left(a_{4}, h_{6}\right),\left(a_{5}, h_{3}\right),\left(a_{6}, h_{5}\right),\left(a_{7}, h_{7}\right),\left(a_{8}, h_{8}\right)\right\}
\end{aligned}
$$

Note that agents $a_{3}$ and $a_{6}$ are indifferent between both assignments, agents $a_{1}, a_{2}, a_{7}$ and $a_{8}$ prefer $\mu^{1}$ to $\mu^{2}$, while agents $a_{4}$ and $a_{5}$ prefer $\mu^{2}$ to $\mu^{1}$. The TTAS mechanisms select one assignment or the other depending on the priority ranking selected at step $0: \sigma^{\succ}=\mu^{2}$ if $\omega_{4} \succ \omega_{1}$ and $\omega_{5} \succ \omega_{7}$; and $\sigma^{\succ}=\mu^{1}$, otherwise. Let us analyze the individual assignment obtained for each agent depending on the selected priority ranking: Consider agents $a_{1}, a_{2}$ and $a_{4} .{ }^{8}$ The utility attained by agents $a_{1}$ and $a_{4}$ depends on the position of their initial endowments in the priority ranking relative to each other, not on their absolute positions: If $\omega_{1}$ is higher up in the ranking than $\omega_{4}$, the mechanism provides $\mu^{1}$. If, however, $\omega_{4}$ is higher up, then their utility will depend on the position of $\omega_{5}$ and $\omega_{7}$ in the priority ranking relative to each other. The case of agent $a_{2}$ is more curious, her utility is not affected by the position of $\omega_{2}$ in the priority ranking, her fate is totally linked to agent $a_{1}$ 's fate.

We have so far presented our family of mechanisms as deterministic, by assuming that the selection of the priority ranking may be performed in terms of some characteristics of the agents (income, seniority, ...). However, if there is no intuitive way of selecting a priority ranking for a particular problem, the possibility of randomizing could be considered. In this case, independently of the probability distribution

${ }^{8}$ Observe that agents $a_{7}, a_{8}$ and $a_{5}$ are in dual situations with respect to these agents, and that $a_{3}$ and $a_{6}$ are not affected by the priority ranking. 
over the priority rankings, we have that the random mechanism thus obtained satisfies individual rationality, strategy-proofness and ex-post efficiency ${ }^{9}$. Additionally, it provides a strict core assignment with probability 1 if the strict core is non-empty and, in general, it provides a core assignment with probability 1.

\subsection{Applications and treatment of indifferences}

Apart from the housing market problem, there are some other interesting problems in the literature that can be seen as the exchange of indivisible goods. Some examples are house allocation with existing tenants (Abdulkadiroğlu and Sönmez (1999)), kidney exchange (Roth et al.(2004)) and school choice (Abdulkadiroğlu and Sönmez (2003)). In order to solve these problems, it is generally assumed that agents have strict preferences and mechanisms adapted from the TTC have been proposed. However, in all these problems, it is natural to think that agents may have indifferences. Therefore, it is also necessary to propose a mechanism in which they can report them. We can easily adapt our family of TTAS mechanisms to each of the problems mentioned above, similarly to the way in which the original TTC is adapted to incorporate the particular characteristics of each of these frameworks. ${ }^{10}$ Therefore, there is a wide range of problems in which our proposed family of mechanisms can be applied.

Regarding the school choice problem, other mechanisms different from the TTC have also been proposed for the case of strict preferences. Abdulkadiroğlu and Sönmez (2003) propose a mechanism based on the Gale-Shapley Deferred Acceptance (DA) algorithm for two-sided matching problems (see Gale and Shapley (1962)), which is the mechanism chosen by some US city authorities to be applied. Erdil and Ergin (2008) propose a generalization of this mechanism for the case in which schools have ties in their priority rankings. They apply the DA algorithm with a particular tie-breaking of the school priorities, and then proceed with the exchange of the slots in the schools in a similar way as the TTC or our TTAS do. The main difference is that they can select any cycle in the original digraph to guarantee strategy-proofness, given that the priorities of the schools (the side of the market that has ties in the rankings in their model) are objective. Then, there is no possibility of manipulation in this side of the matching market. On the contrary, in our model the ties are in agents' preferences and, as a consequence, the selection of the cycles in the digraph cannot be arbitrary to maintain strategy-proofness. Both the search for absorbing sets and the inclusion of the priority ranking to select cycles are necessary to maintain strategy-proofness in our model.

\subsection{Computational complexity}

From a practical point of view, it is interesting to analyze the computational complexity of any mechanism. We next discuss some details on the computational complexity of the TTAS algorithm, in which the TTAS mechanisms are based.

Steps from (i.1) to (i.3) of the algorithm, which construct the graph, calculate the absorbing sets and classify if they are paired-symmetric or not, can be computed in polynomial time. In fact, the analysis of the computational complexity of these parts would be similar to the analysis of the algorithm to compute the "partition by minimal self-mapped sets" introduced by Quint and Wako (2004), which is polynomial of

\footnotetext{
${ }^{9}$ This is a difference with respect to the mechanism proposed by Yllmaz (2009), which is ex-ante efficient, but not strategyproof.

${ }^{10}$ The particular details of the adaptations can be provided upon request.
} 
degree $3 .^{11}$

Unfortunately, step (i.4) may create a computational handicap, as with the selection of cycles proposed in this step, it could be the case that in a sequence of iterations in a non paired-symmetric absorbing set nobody obtains a better house than the one owned at step $i$, in such a way that the formation of paired-symmetric absorbing sets would be retarded during a number of steps. In case that this fact substantionally increases the complexity of the TTAS algorithm, it would be interesting to redesign this step (i.4) in a computationally more efficient way.

\section{APPENDIX}

The proofs for all the results of the paper are set out in the Appendix.

\section{Proof of Proposition 1}

By contradiction, suppose that this does not occur. That is, there is a maximal set of individuals $S \subseteq N$ and a maximal set of houses $T \subseteq H$ that are never removed from the algorithm. Consider the algorithm from the step immediately subsequent to the removal of any other agent and house, say step $i_{1}$. Some characteristics of this step and its relation with step $i_{1}+1$ are the following:

- In the digraph of step $i_{1}$, we know that there is at least one absorbing set, and all of them are non paired-symmetric, because if an absorbing set is paired-symmetric, then its agents and houses would leave the algorithm and we would have a contradiction. Therefore, a set of nodes not belonging to symmetric pairs exists in each absorbing set. Hence, at step $i_{1} .3$, the algorithm selects one house for each agent to point to, obtaining a subgraph for each absorbing set with some cycles. Next, at step $i_{1} .4$, the algorithm proceeds to assign provisionally the houses in the cycles of the subgraph to the agents pointing to them.

- Consider any node $v$ of an absorbing set at step $i_{1}$ and any other node $v^{\prime}$ not belonging to this absorbing set. Then, $v$ and $v^{\prime}$ cannot belong to the same absorbing set at step $i_{1}+1$. This happens because any agent or house does not leave the market at step $i_{1}$. Hence, if $v$ is a node representing an agent, then at step $i_{1}+1, v$ points to the same houses as at step $i_{1}$. If $v$ is a node representing a house, either $v$ will point at step $i_{1}+1$ to the same agent as at step $i_{1}$ if it has not entered a selected cycle at step $i_{1} .4$, or it will point to other agent belonging to the absorbing set if it has entered a selected cycle. Therefore, no absorbing set at step $i_{1}$ increases its set of nodes from this step on.

Additionally, notice that each absorbing set of any step $t>i_{1}$ is a (non-necessarily strict) subset of an absorbing set of step $i_{1}$. Given that we have assumed that the nodes of these absorbing sets of step $i_{1}$ always keep in the algorithm, we have that there should be a step, say $i_{2}$, from which the set of nodes in any absorbing set will never decrease. Consider each absorbing set $A=S_{A} \cup T_{A} \subseteq S \cup T$ at this step, and let $S_{A}^{\prime} \cup T_{A}^{\prime} \subseteq A$ be the set of nodes not belonging to any symmetric pairs. Then, it is clear that the nodes of $A \backslash\left(S_{A}^{\prime} \cup T_{A}^{\prime}\right)$ will also belong to symmetric pairs in step $i_{2}+1$. With respect to the nodes of $S_{A}^{\prime} \cup T_{A}^{\prime}$, each of them will belong to a symmetric pair in step $i_{2}+1$ only if it has entered a selected cycle at step

\footnotetext{
${ }^{11}$ For example, the computation of the absorbing sets of a graph would be based in Tarjan's (1972) algorithm, which is linear.
} 
$i_{2} .4$. Therefore, the set of nodes of an absorbing set $A$ at step $i_{2}$ that belong to symmetric pairs is never decreasing. Note that if for any step $j>i_{2}$, there exists a step $k$, with $k>j$, such that the set of nodes of the absorbing set $A$ belonging to symmetric pairs at step $k$ is bigger than at step $j$, then it is easy to see that we would end up obtaining a paired-symmetric absorbing set, some agents and houses would leave the algorithm and we would reach a contradiction. Therefore, there must be a step, say step $i_{3}$, from which the set of nodes of the absorbing set $A$ that do not belong to any symmetric pair is stable over time. Let this set be $\bar{S}_{A} \cup \bar{T}_{A} \subseteq S_{A}^{\prime} \cup T_{A}^{\prime}$. Some characteristics of this set are the following:

- Each agent of $\bar{S}_{A}$ possesses her initial endowment in all steps, because she has not previously entered any selected cycle. Additionally, this initial endowment is not one of her maximal houses among the ones in the set $T$, since she does not belong to any symmetric pair.

- After applying the priority criterion to select one house for each agent to point to in the absorbing sets at step $i_{3} .3$, the nodes in $\bar{S}_{A} \cup \bar{T}_{A}$ are partitioned in various chains. All these chains finish in a house in $T_{A} \backslash \bar{T}_{A}$ because otherwise, the nodes in $\bar{S}_{A} \cup \bar{T}_{A}$ outside the chains would have formed a cycle at step $i_{3} .4$ and they would belong to some symmetric pairs at step $i_{3}+1$. Note that these chains will never break from this step on because the priority criterion applied at any step $j .3$, with $j \geq i_{3}$, will always select the same house for each of these agents, given that they will never enter a selected cycle.

Given that the agents of $\bar{S}_{A}$ belong to an absorbing set with the agents of $S_{A} \backslash \bar{S}_{A}$, there exists a maximal non-empty set of agents of $S_{A} \backslash \bar{S}_{A}$, denoted by $B_{S}\left(\bar{T}_{A}\right) \subseteq S_{A} \backslash \bar{S}_{A}$, who point to some houses in $\bar{T}_{A}$ at step $i_{3} .1$.

Next, we will show that there must exist a step, say $i_{4}$, from when no nodes in $B_{S}\left(\bar{T}_{A}\right)$ will enter any cycle selected by the algorithm. Suppose that an agent $i \in B_{S}\left(\bar{T}_{A}\right)$ enters selected cycles occasionally from step $i_{3}$ on without end. Then, there must be a step $l$ in which the priority criterion selects a house belonging to an agent in $\bar{S}_{A}$, that is, a house in $\bar{T}_{A}$. Then, this agent $i$ will never enter a selected cycle at any step from $l$ on, because her entry would imply the entry of the agent in $\bar{S}_{A}$, which is a contradiction.

Then, we have that from step $i_{4}$ on, the set of agents of $B_{S}\left(\bar{T}_{A}\right)$ never enter a cycle selected by the algorithm. Let $B_{T}\left(\bar{T}_{A}\right)$ denote the set of houses that the agents in $B_{S}\left(\bar{T}_{A}\right)$ possess at step $i_{4}$. Note that after applying the priority criterion at step $i_{4} .3$, the nodes in the set $\bar{S}_{A} \cup \bar{T}_{A} \cup B_{S}\left(\bar{T}_{A}\right) \cup B_{T}\left(\bar{T}_{A}\right)$ are partitioned in various chains finishing in houses in the set $T_{A} \backslash\left(\bar{T}_{A} \cup B_{T}\left(\bar{T}_{A}\right)\right)$, which will never break from this step on. If $B_{S}\left(\bar{T}_{A}\right) \cup B_{T}\left(\bar{T}_{A}\right) \cup \bar{S}_{A} \cup \bar{T}_{A}=A$, we could conclude that no node enters cycles selected by the algorithm from this step on. However, this is not possible since the algorithm always selects at least one cycle from each absorbing set which is not paired-symmetric. Then, we have that $B_{S}\left(\bar{T}_{A}\right) \cup B_{T}\left(\bar{T}_{A}\right) \cup \bar{S}_{A} \cup \bar{T}_{A} \neq A$. Given that the nodes of $B_{S}\left(\bar{T}_{A}\right) \cup B_{T}\left(\bar{T}_{A}\right) \cup \bar{S}_{A} \cup \bar{T}_{A}$ belongs to an absorbing set with the other nodes of $A$, there exists a maximal non-empty set of agents of $S_{A} \backslash\left(\bar{S}_{A} \cup B_{S}\left(\bar{T}_{A}\right)\right)$, denoted by $B_{S}\left(B_{T}\left(\bar{T}_{A}\right)\right) \subseteq S_{A} \backslash\left(\bar{S}_{A} \cup B_{S}\left(\bar{T}_{A}\right)\right)$, who point to some houses in $B_{T}\left(\bar{T}_{A}\right)$ at step $i_{4} .1$. Then, by applying the same reasoning as before, we can deduce that there exists a step $i_{5}$ such that the agents in $B_{S}\left(B_{T}\left(\bar{T}_{A}\right)\right)$ (and their current houses) never enter selected cycles from this step on. Applying recursively this argument, and given that absorbing sets have a finite set of nodes, we will finally conclude that there is a step $i_{m}$ such that no node enters selected cycles from this step on, given that all nodes of $A$ belong to some set $B_{S}\left(B_{T}\left(B_{T}\left(\ldots\left(B_{T}\left(\bar{T}_{A}\right)\right)\right)\right)\right)$ or $B_{T}\left(B_{S}\left(B_{T}\left(B_{T}\left(\ldots\left(B_{T}\left(\bar{T}_{A}\right)\right)\right)\right)\right)\right.$. However, this is not possible since cycles are selected in all steps of the algorithm for each absorbing set which is not paired-symmetric. Therefore, we have reached a contradiction and the proposition is proved. 


\section{An important lemma}

We include a lemma which will be helpful in the proof of Theorem 1. This lemma states that an agent will be indifferent towards all the houses assigned to her provisionally by the TTAS algorithm.

Lemma 1 Let $\mu_{i}^{t}$ be the $t$-th house provisionally assigned by the TTAS algorithm to agent $i$. Then, $\mu_{i}^{t} I_{i} \mu_{i}^{t+1}$ for all $t$.

Proof. Consider the step of the algorithm at which $\mu_{i}^{t+1}$ is assigned to agent $i$ and let $\mu_{i}^{t}$ be agent $i$ 's previous assignment. Then, by construction of the algorithm, there is a selected cycle at substep 4 in which $\mu_{i}^{t}$ points to $i$ and $i$ points to $\mu_{i}^{t+1}$. Therefore, $\mu_{i}^{t+1}$ is a maximal house for $i$ among the ones remaining in the algorithm at this step. Given that $\mu_{i}^{t}$ is in the algorithm at this step, $\mu_{i}^{t+1} R_{i} \mu_{i}^{t}$.

On the other hand, when previously $\mu_{i}^{t}$ was provisionally assigned to $i$ by the algorithm, $\mu_{i}^{t+1}$ was in the algorithm as well. Therefore, since at that step $\mu_{i}^{t}$ was a maximal house for $i, \mu_{i}^{t} R_{i} \mu_{i}^{t+1}$.

Thus, we can conclude that $\mu_{i}^{t} I_{i} \mu_{i}^{t+1}$, as desired.

From this lemma, we can deduce the following corollary which will be useful for proving subsequent results. It says that the first house that the TTAS algorithm assigns provisionally to an agent determines the utility that this agent will obtain from her final assignment.

Corollary 2 Let $\sigma^{\succ}(i)$ be the assignment that the TTAS mechanism with priority ranking $\succ$ assigns to agent $i$ and let $\mu_{i}^{1}(\succ)$ be the first provisional assignment (different from the initial endowment) that the TTAS algorithm with this priority ranking assigns to agent $i$. Then, $\sigma^{\succ}(i) I_{i} \mu_{i}^{1}(\succ)$.

\section{Proof of Theorem 1}

By contradiction, suppose that there is some TTAS mechanism $\sigma^{\succ}$ that selects for some housing market problem $(N, H, \omega, R)$ an assignment $\mu$ which is not in the core. Then, a coalition $T \subseteq N$ and an assignment $v$ exist such that for all $i \in T, v_{i} \in \omega(T)$ and $v_{i} P_{i} \mu_{i}$. Denote, without loss of generality, $T=\{1,2, \ldots, r\}$ such that for all $i \in\{1, \ldots, r\} v_{i}=\omega_{i+1}$ (subscript modulo $r$ ). Consider agent 1. Given that $v_{1} P_{1} \mu_{1}$, we have that $v_{1}$ has left the algorithm before agent 1 . Then, $\omega^{-1}\left(v_{1}\right)=2 \in T$ has entered a selected cycle and received a provisional assignment before agent 1 . Moreover, since $v_{2} P_{2} \mu_{2}$, this implies that $v_{2}$ has left the algorithm before agent 2. Then, $\omega^{-1}\left(v_{2}\right)=3 \in T$ has entered a selected cycle and received a provisional assignment before agent 2 and so on. Following this argument, we have that for all $i \in T, v_{i}$ has left the market before agent $i$ and that agent $i+1=\omega^{-1}\left(v_{i}\right)(i$ modulo $r$ ) has entered a selected cycle and received a provisional assignment before agent $i$. Then, we conclude that agent 1 has entered a selected cycle and received a provisional assignment before agent 1 , which is a contradiction. Then, the theorem is proved.

\section{Proof of Theorem 2}

By contradiction, suppose that there is some TTAS mechanism $\sigma^{\succ}$ that selects for some housing market problem $(N, H, \omega, R)$ an assignment $\mu$ which is not Pareto efficient. That is, an assignment $\nu$ exists such that, for all $i \in N, \nu_{i} R_{i} \mu_{i}$ and for some $j \in N, \nu_{j} P_{j} \mu_{j}$. By construction of the algorithm, $\nu_{j}$ entered a paired-symmetric absorbing set, say $A_{l}^{*}$, with agent $\mu^{-1}\left(\nu_{j}\right)$ and they left the algorithm before agent $j$ and $\mu_{j}$. Without loss of generality, suppose that $\mu^{-1}\left(\nu_{j}\right)=j+1$. Then, by Pareto dominance of $\nu$ over 
$\mu, \nu_{j+1} R_{j+1} \nu_{j}$. Now, we prove that at least one agent of $A_{l}^{*}$ strictly prefers her assignment in $\nu$ to her assignment in $\mu$. If $j+1$ is not this individual and $\nu_{j+1} I_{j+1} \nu_{j}$, then $\nu_{j+1}$ belongs to $A_{l}^{*}$ jointly with agent $\mu^{-1}\left(\nu_{j+1}\right)$. Therefore, applying recursively this argument we can deduce that some agent in $A_{l}^{*}$ must prefer strictly her assignment in $\nu$ to her assignment in $\mu$, because otherwise agent $j$ would be in $A_{l}^{*}$ and this is a contradiction.

Then, let $k$ be an agent in $A_{l}^{*}$ such that $\nu_{k} P_{k} \mu_{k}$. Then, following the same analysis as before, $\nu_{k}$ entered a paired-symmetric absorbing set, say $A_{l-1}^{*}$ with agent $\mu^{-1}\left(\nu_{k}\right)$ at a previous step and there is some agent in $A_{l-1}^{*}$ strictly preferring her assignment in $\nu$ to her assignment in $\mu$. If we apply recursively the previous analysis, given that this process is finite, we arrive at the first paired-symmetric absorbing set $A_{1}^{*}$ and we will not be able to continue further. Therefore, we have a contradiction and the theorem is proved.

\section{Proof of Theorem 3}

We prove some lemmas that will help us in the proof of the theorem.

Hereafter, we will denote by $\sigma^{\succ}\left(R_{-i}, R_{i}\right)$ the TTAS mechanism with the priority ranking $\succ$ when the reported preferences are $\left(R_{-i}, R_{i}\right)$ and the description of $N, H$ and $\omega$ is clear.

Lemma 2 Let $h_{k}$ be the first house, different from the initial endowment, assigned provisionally to agent $i$ by the TTAS algorithm defining $\varphi^{\succ}\left(R_{-i}, R_{i}\right)$ and let $R_{i}^{\prime}$ be any preference such that $\left\{h \in H \mid h P_{i} h_{k}\right\}=\{h \in$ $\left.H \mid h P_{i}^{\prime} h_{k}\right\}$ and there does not exist $h \in H \backslash\left\{h_{k}\right\}$ such that $h I_{i}^{\prime} h_{k}$. Then,

(i) $h_{k}$ is also the first house, different from the initial endowment, assigned provisionally to agent $i$ by the TTAS algorithm defining $\varphi^{\succ}\left(R_{-i}, R_{i}^{\prime}\right)$, and

(ii) the set of selected cycles and paired-symmetric absorbing sets preceding the cycle assigning $h_{k}$ to agent $i$ by the TTAS algorithm defining $\varphi^{\succ}\left(R_{-i}, R_{i}\right)$ are also selected by the TTAS algorithm defining $\varphi^{\succ}\left(R_{-i}, R_{i}^{\prime}\right)$ before the selection of the cycle assigning $h_{k}$ to agent $i$.

Proof. Let $h_{k}$ and agent $i$ enter jointly for first time a selected cycle by the algorithm defining $\varphi^{\succ}\left(R_{-i}, R_{i}\right)$ at step $q$.

Let $t=1$ be the first step of the algorithm and let $G_{1}^{\succ}\left(R_{-i}, R_{i}\right)$ be the digraph associated with this step when agent $i$ declares $R_{i}$. Suppose that $q>1$ (otherwise, the proof is finished). Notice that $G_{1}^{\succ}\left(R_{-i}, R_{i}\right)$ and $G_{1}^{\succ}\left(R_{-i}, R_{i}^{\prime}\right)$ only differ from each other in the arcs leading from agent $i$ and therefore every pairedsymmetric absorbing set in $G_{1}^{\succ}\left(R_{-i}, R_{i}\right)$ is also in $G_{1}^{\succ}\left(R_{-i}, R_{i}^{\prime}\right)$. Let $c_{j}=\left\{1, h_{2}, 2, h_{3}, \ldots, h_{1}\right\}$ be a cycle selected by the algorithm at the end of this step. Now, consider $G_{1}^{\succ}\left(R_{-i}, R_{i}^{\prime}\right)$ and note that every node in $c_{j}$ is in this graph pointing to the same nodes as in $G_{1}^{\succ}\left(R_{-i}, R_{i}\right)$ (given that $i \notin c_{j}$ ).

(a) If $c_{j}$ is in an absorbing set in $G_{1}^{\succ}\left(R_{-i}, R_{i}^{\prime}\right)$, all the houses pointed by agents in $c_{j}$ in $G_{1}^{\succ}\left(R_{-i}, R_{i}\right)$ are also in this absorbing set. Then, the same structure of priorities is used to select an arc from each agent of $c_{j}$ in $G_{1}^{\succ}\left(R_{-i}, R_{i}\right)$ and in $G_{1}^{\succ}\left(R_{-i}, R_{i}^{\prime}\right)$. Hence, $c_{j}$ is also a selected cycle at the first step of the algorithm for $\left(R_{-i}, R_{i}^{\prime}\right)$.

(b) Otherwise, all agents and houses in $c_{j}$ will enter an absorbing set (the same for all of them) for the first time at the same step of the algorithm. Then, at this step, say step $t$, although the absorbing set could be different from the one to which $c_{j}$ belongs in $G_{1}\left(R_{-i}, R_{i}\right)$, the structure of priorities will give the same result as in $G_{1}^{\succ}\left(R_{-i}, R_{i}\right)$ for the agents of $c_{j}$. Thus, the cycle $c_{j}$ will be also selected in $G_{t}^{\succ}\left(R_{-i}, R_{i}^{\prime}\right)$. 
Additionally, notice that this is also the first selected cycle where the nodes in $c_{j}$ enter when the preferences are $\left(R_{-i}, R_{i}^{\prime}\right)$.

Let now $t=2$ and and let $G_{2}^{\succ}\left(R_{-i}, R_{i}\right)$ be the digraph associated with this step when agent $i$ declares $R_{i}$. Suppose that $q>2$ (otherwise, the proof is finished). Notice that $G_{1}^{\succ}\left(R_{-i}, R_{i}\right)$ and $G_{1}^{\succ}\left(R_{-i}, R_{i}^{\prime}\right)$ have the same paired-symmetric absorbing sets and, then, the nodes removed at the first step of the algorithm are the same in both graphs.

First, we will show that every paired-symmetric absorbing set in $G_{2}^{\succ}\left(R_{-i}, R_{i}\right)$ is also obtained by the algorithm when preferences are $\left(R_{-i}, R_{i}^{\prime}\right)$. Consider any paired-symmetric absorbing set, $A_{i}^{*}$, in $G_{2}^{\succ}\left(R_{-i}, R_{i}\right)$. It is clear that every arc leading from an agent in $A_{i}^{*}$ in $G_{2}^{\succ}\left(R_{-i}, R_{i}\right)$ also appears in $G_{2}^{\succ}\left(R_{-i}, R_{i}^{\prime}\right)$ and at subsequent steps, while this agent and the houses she is pointing to are in the algorithm.

Consider a house $h_{m} \in A_{i}^{*}$. Suppose first that $h_{m}$ points to its original owner $m$ in $A_{i}^{*}$. This means that $h_{m}$ does not enter a selected cycle in the first step of the algorithm when the preferences are $\left(R_{-i}, R_{i}\right)$. Then, we are going to show that it will also point to $m$ in $G_{2}^{\succ}\left(R_{-i}, R_{i}^{\prime}\right)$. By contradiction, suppose that this does not occur. Then, $h_{m}$ and $m$ entered a selected cycle in the first step of the algorithm when the preferences are $\left(R_{-i}, R_{i}^{\prime}\right)$. Then, by the same argument as before, the cycles selected in the first step of the algorithm when the preferences are $\left(R_{-i}, R_{i}^{\prime}\right)$ will appear in some step $t$ when the preferences are $\left(R_{-i}, R_{i}\right)$ and this will be the first selected cycle in which these nodes enter. In particular, this will be the first selected cycle in which $h_{m}$ and $m$ enter when the preferences are $\left(R_{-i}, R_{i}\right)$. But this is a contradiction since $h_{m}$ and $m$ abandon the algorithm in the second step when the preferences are $\left(R_{-i}, R_{i}\right)$ without entering a selected cycle. Then each house in $A_{i}^{*}$ pointing to its original owner in $G_{2}^{\succ}\left(R_{-i}, R_{i}\right)$ will also point to her in $G_{2}^{\succ}\left(R_{-i}, R_{i}^{\prime}\right)$ and at subsequent steps, while this house and its owner are in the algorithm.

Consider now a house $h_{m}$ that points in $A_{i}^{*}$ to an agent $j$ different from its original owner in $G_{2}^{\succ}\left(R_{-i}, R_{i}\right)$. Then, $h_{m}$ must belong to a cycle $c_{j}$ selected in $G_{1}^{\succ}\left(R_{-i}, R_{i}\right)$. Then, by the previous reasoning, we know that $c_{j}$ will also be selected in $G_{t}^{\succ}\left(R_{-i}, R_{i}^{\prime}\right)$ for some $t$ and, then, $h_{m}$ points to $j$ in $G_{t+1}\left(R_{-i}, R_{i}^{\prime}\right)$.

Therefore, the paired-symmetric absorbing set $A_{i}^{*}$ will be obtained in $G_{t^{*}+1}^{\succ}\left(R_{-i}, R_{i}^{\prime}\right)$, where $t^{*}$ is the later step at which a house in $A_{i}^{*}$ has entered its corresponding selected cycle ${ }^{12}$. And, therefore, the sequence of provisional assignments that has received each of these agents are the same in both cases.

Now we will show that every cycle selected from a non paired-symmetric absorbing set in $G_{2}^{\succ}\left(R_{-i}, R_{i}\right)$ is also selected when the declared preference is $\left(R_{-i}, R_{i}^{\prime}\right)$. Let $c_{j}=\left\{a_{1}, h_{2}, a_{2}, h_{3}, \ldots, h_{1}\right\}$ be a selected cycle in $G_{2}^{\succ}\left(R_{-1}, R_{i}\right)$. As we have mentioned before, $G_{2}^{\succ}\left(R_{-i}, R_{i}\right)$ and in $G_{2}^{\succ}\left(R_{-i}, R_{i}^{\prime}\right)$ have the same set of nodes and, therefore, the agents of $c_{j}$ point to the same houses in both graphs.

Consider a house $h_{m} \in c_{j}$. Suppose first that $h_{m}$ points to its original owner $m$ in $c_{j}$. This means that $h_{m}$ does not enter a selected cycle in the first step of the algorithm when the preferences are $\left(R_{-i}, R_{i}\right)$. Then, we are going to prove that either $h_{m}$ points to $m$ in $G_{2}^{\succ}\left(R_{-i}, R_{i}^{\prime}\right)$ or $c_{j}$ is a selected cycle in $G_{1}^{\succ}\left(R_{-i}, R_{i}^{\prime}\right)$. In order to prove it, suppose that $h_{m}$ does not point to $m$ in $G_{2}^{\succ}\left(R_{-i}, R_{i}^{\prime}\right)$. Then, $h_{m}$ and $m$ enter together a selected cycle for first time in $G_{1}^{\succ}\left(R_{-i}, R_{i}^{\prime}\right)$. By the same argument as before, the cycles selected in the first step of the algorithm when the preferences are $\left(R_{-i}, R_{i}^{\prime}\right)$ appear for first time in some step $t$ when the preferences are $\left(R_{-i}, R_{i}\right)$. In this case, $t=2$ by the previous assumption. Then, we have that $c_{j}$ is

\footnotetext{
${ }^{12}$ If all of them point to their original owner, $t^{*}=1$
} 
selected in the first step of the algorithm when the preferences are $\left(R_{-i}, R_{i}^{\prime}\right)$ and the sequence of provisional assignments to each of the agents in $c_{j}$ is the same in both cases.

Consider now a house $h_{m} \in c_{j}$ that points to an agent $j$ different from its original owner. Then, $h_{m}$ must belong to a cycle $c_{k}$ selected in $G_{1}^{\succ}\left(R_{-i}, R_{i}\right)$. Then, by the previous reasoning, we know that $c_{k}$ will also be selected in $G_{t}^{\succ}\left(R_{-i}, R_{i}^{\prime}\right)$ for some $t$. Let $t^{*}$ be the later step at which one of these selected cycles is formed when the preferences are $\left(R_{-i}, R_{i}^{\prime}\right)$ ( $t^{*}=1$ if every house points to its initial owner). Then, $G_{2}^{\succ}\left(R_{-i}, R_{i}\right)\left|c_{j}=G_{t^{*}+1}^{\succ}\left(R_{-i}, R_{i}^{\prime}\right)\right| c_{j}{ }^{13}$. Then, we also have that there exists $\hat{t} \geq t^{*}+1$ such that $G_{2}^{\succ}\left(R_{-i}, R_{i}\right)\left|c_{j}=G_{\hat{t}}^{\succ}\left(R_{-i}, R_{i}^{\prime}\right)\right| c_{j}$ and $c_{j}$ belongs to some absorbing set in both graphs. Given that $c_{j}$ is formed in $G_{2}^{\succ}\left(R_{-i}, R_{i}\right)$, we can deduce that the house $h_{j}$ provisionally assigned to each agent $l$ in $c_{j}$ at this step is the house with the highest priority between the set of maximal houses of $l$ among the remaining ones excluding their previous provisional assignments. It is possible that in $G_{\hat{t}}^{\succ}\left(R_{-i}, R_{i}^{\prime}\right)$ the set of maximal houses of agent $l$ among the remaining houses is a proper subset of that in $G_{2}^{\succ}\left(R_{-i}, R_{i}\right)$, but $h_{j}$ is still present and the sequence of provisional assignments of agent $l$ is the same in both cases. Therefore, the priority criterion will also select the arc from agent $l$ to house $h_{j}$ in $G_{\hat{t}}^{\succ}\left(R_{-i}, R_{i}^{\prime}\right)$. Then, $c_{j}$ is also selected in step $\hat{t}$ and the sequence of provisional assignments to each of these agents is the same in both cases.

When $t \in\{3, \ldots, q\}$, the proof can be performed with similar reasonings. Then, we have proved that the set of selected cycles and paired-symmetric absorbing sets preceding the cycle assigning $h_{k}$ to agent $i$ by the TTAS algorithm defining $\varphi^{\succ}\left(R_{-i}, R_{i}\right)$ are also selected by the TTAS algorithm defining $\varphi^{\succ}\left(R_{-i}, R_{i}^{\prime}\right)$ before the selection of the cycle assigning $h_{k}$ to agent $i$ (part (ii) of the lemma).

Now, we have to prove that $h_{k}$ is also the first house, different from the initial endowment, assigned provisionally to agent $i$ by the TTAS algorithm defining $\varphi^{\succ}\left(R_{-i}, R_{i}^{\prime}\right)$ (part $(i)$ of the lemma). We know, by part $(i i)$, that the sequence of provisional assignments to each agent in the first $q$ steps of the TTAS algorithm defining $\varphi^{\succ}\left(R_{-i}, R_{i}\right)$ is the same that the sequence of provisional assignments to each agent in the first $v$ steps of the TTAS algorithm defining $\varphi^{\succ}\left(R_{-i}, R_{i}^{\prime}\right)$. Additionally, the nodes that have disappeared from the algorithm are also the same. Then, agent $i$ enters the same absorbing set in step $q$ of the TTAS algorithm defining $\varphi^{\succ}\left(R_{-i}, R_{i}\right)$ and in step $v$ of the TTAS algorithm defining $\varphi^{\succ}\left(R_{-i}, R_{i}^{\prime}\right)$, and the priority criterion obtains the same result in both cases. Then, $h_{k}$ is also the first house assigned provisionally to $i$ when the preferences are $\left(R_{-i}, R_{i}^{\prime}\right)$ and the lemma is proved.

Now, we prove that, if an agent attains a utility level declaring a preference $R_{i}$, then there exists a house which gives this agent the same utility and the mechanism will assign it to her if she makes it her maximal house.

Lemma 3 Let $U_{i}\left(\varphi_{i}^{\succ}\left(R_{-i}, R_{i}\right)\right)=k$, then there exists a house $h_{j}$ such that $U_{i}\left(h_{j}\right)=k$ and $\varphi_{i}^{\succ}\left(R_{-i}, R_{i}^{\prime}\right)=h_{j}$ for all $R_{i}^{\prime}$ with $\max _{H}\left(R_{i}^{\prime}\right)=\left\{h_{j}\right\}$.

Proof. Let $h_{j}$ be the first house assigned provisionally to agent $i$ by the TTAS algorithm for $\left(R_{-i}, R_{i}\right)$ when the priority ranking is $\succ$. Notice that, by Corollary $2, U_{i}\left(h_{j}\right)=U_{i}\left(\varphi_{i}^{\succ}\left(R_{-i}, R_{i}\right)=k\right.$. Following the same reasoning as in the proof of Lemma 2 , we can conclude that the absorbing set of agent $i$ in the graph corresponding to step $q$ of the algorithm in which $h_{j}$ is assigned to $i$ when she declares $R_{i}$ is the same as

\footnotetext{
${ }^{13}$ If $G$ is a graph and $c$ is a set of nodes of $G$, we denote by $G \mid c$ the restricted graph that includes only the nodes of $c$ and the arrows leading from a node of $c$ to another node of $c$.
} 
the absorbing set of agent $i$ in the graph corresponding to step $v$ of the algorithm when she declares $R_{i}^{\prime}$. Additionally, we know that each agent has passed from the same sequence of provisional assignments in both algorithms until these steps are reached. Then, if the priority criterion has selected the cycle in which $h_{j}$ is assigned to $i$ when she declares $R_{i}$, the priority criterion has to select this cycle also when she declares $R_{i}^{\prime}$. Given that $\max _{H}\left(R_{i}^{\prime}\right)=\left\{h_{j}\right\}$, we have, by Corollary 2 , that $\varphi_{i}^{\succ}\left(R_{-i}, R_{i}^{\prime}\right)=h_{j}$.

Now, we can prove the theorem. By Lemma 3, we have that, if there is any way of obtaining a particular level of utility, the same level can also be obtained by declaring any preference in which the maximal house is one of the houses $\left(h_{k}\right)$ that provides this utility. Let $h_{l}$ be the first house assigned provisionally to agent $i$ by the TTAS algorithm defining $\varphi^{\succ}\left(R_{-i}, R_{i}\right)$. By Corollary 2 , we have $\varphi_{i}^{\succ}\left(R_{-i}, R_{i}\right) I_{i} h_{l}$. Now assume that a house $h_{k}$ with $h_{k} P_{i} h_{l}$ can be assigned to agent $i$ by her declaring some modified preference. Pick any ranking $R_{i}^{\prime}$ such that (i) $\max _{H}\left(R_{i}^{\prime}\right)=\left\{h_{k}\right\}$, (ii) $\left\{h \in H \mid h P_{i} h_{l}\right\}=\left\{h \in H \mid h P_{i}^{\prime} h_{l}\right\}$ and there does not exist $h \in H \backslash\left\{h_{l}\right\}$ with $h I_{i}^{\prime} h_{l}$. Then, by (i) and Lemma 3, we have $\varphi_{i}^{\succ}\left(R_{i}, R_{i}^{\prime}\right)=h_{k}$. On the other hand, from (ii), Corollary 2 and Lemma 2, we have $\varphi_{i}^{\succ}\left(R_{-i}, R_{i}^{\prime}\right) I_{i} h_{l}$. However, this is impossible, since $h_{k} P_{i} h_{l}$. Therefore, the theorem is proved.

\section{Proof of Theorem 4}

Consider a housing market problem $(N, H, \omega, R)$ with a non-empty strict core. We need to introduce an algorithm, called Top Trading Segmentation originally proposed by Quint and Wako (2004), which provides a partition of the set of agents and houses which is useful to determine if the strict-core is non-empty, and in that case, to provide an assignment in the strict core of the problem.

The Top Trading Segmentation (TTS) algorithm

Step 1: Let each agent point to her maximal houses and each house point to its owner. Select the absorbing sets of this digraph. Each absorbing set constitutes a set of the partition.

Step i: Let each agent point to her maximal houses among the remaining ones and each remaining house point to its owner. Select the absorbing sets of this digraph. Each absorbing set constitutes a set of the partition.

With the partition obtained with the algorithm, Quint and Wako (2004) prove that the following statements are equivalent:

- In each set of the partition, it is possible to find a sub-allocation that assigns to each agent one of her maximal houses in this set.

- The strict core of the problem is non-empty and one of its allocations consists of the union of all these sub-allocations.

Now, we will prove the following lemma.

Lemma 4 Consider a digraph $(V, E)$ such that $V$ is an absorbing set. Then, we can partition $V$ into a set of disjoint cycles if and only if there exists a subset $E^{\prime} \subseteq E$ such that in the subgraph $\left(V, E^{\prime}\right)$ all nodes have indegree and outdegree equal to 1. 
Proof. $(\Rightarrow)$ Assume that we can partition $V$ into a set of disjoint cycles, $\left\{c^{i}=\left(h_{1}^{i}, a_{1}^{i}, h_{2}^{i}, a_{2}^{i}, \ldots, h_{m_{i}}^{i}, a_{m_{i}}^{i}\right)\right\}_{i \in\{1, \ldots, k\}}$. Then, construct $E^{\prime}$ as follows: $(x, y) \in E^{\prime}$ if and only if $\left[x=a_{j}^{i}\right.$ and $\left.y=h_{j+1}^{i}\right]$ (subscript modulo $m_{i}$ ) or $\left[x=h_{j}^{i}\right.$ and $\left.y=a_{j}^{i}\right]$ for some $i \in\{1, \ldots, k\}$ and $j \in\left\{1, \ldots, m_{i}\right\}$. It is easy to see that in the subgraph $\left(V, E^{\prime}\right)$ all nodes have indegree and outdegree equal to 1.

$(\Leftarrow)$ Assume now that there exists a subset $E^{\prime} \subseteq E$ such that in the subgraph $\left(V, E^{\prime}\right)$ all nodes have indegree and outdegree equal to 1 . Take any node of this subgraph and follow the path of edges leading from this node (this path is unique since the outdegree of all nodes is 1). Given that the indegree of all nodes is equal to 1 , this path will terminate at some point at the initial node and it is the first cycle of the partition. Take another node not belonging to the previous cycle and construct another cycle as before. Given that $V$ is finite, following this procedure we will finally have a partition of $V$ into a set of disjoint cycles.

Then, consider the absorbing sets from the first step of the TTAS algorithm. Note that each of these absorbing sets is also one of the sets of the partition determined by the TTS algorithm. Then, given the result of Quint and Wako (2004), we have that in a housing market problem with a non-empty strict core, these absorbing sets can be partitioned into disjoint cycles, which represent strict core sub-allocations. Or, equivalently, using Lemma 4 , for each absorbing set $A_{i}$ at step 1 and the associated graph $\left(A_{i}, E_{A_{i}}\right)$, there is a subgraph $\left(A_{i}, E_{A_{i}}^{\prime}\right)$, such that $E_{A_{i}}^{\prime} \subseteq E_{A_{i}}$ and every node in $A_{i}$ has indegree and outdegree equal to 1.

Let $A_{i}$ be an absorbing set at step 1 of the TTAS algorithm. There are two cases:

Case 1: If $A_{i}$ is paired-symmetric, there is a set of disjoint cycles assigning to each agent one of her maximal houses: each cycle is formed by each agent and her house. Given that in this case the TTAS algorithm assigns to each agent her current house, the assignment obtained matches a strict-core sub-allocation.

Case 2: If $A_{i}$ is not paired-symmetric, by using a priority ranking $\succ$ the algorithm selects one edge for each node in $A_{i}$ at step 1.3. Then, some cycles are formed and the agents in these cycles provisionally exchange their houses at step 1.4. There are two possibilities:

(2.1) If the cycles selected determine a partition of $A_{i}$, then all nodes of $A_{i}$ will form symmetric pairs in the next step and they will leave the algorithm with the houses obtained in these cycles at this next step or at subsequent steps. ${ }^{14}$ Then, this assignment matches a strict core sub-allocation.

(2.2) If the cycles selected, however, do not determine a partition of $A_{i}$, the TTAS algorithm does not provide a provisional assignment that matches a strict core sub-allocation in the first step, but we are going to prove that it will do in the next ones.

On the one hand, the nodes of $A_{i}$ that have entered a selected cycle in step 1 will belong to symmetric pairs in the next step. On the other hand, the remaining nodes of $A_{i}$ will belong to some absorbing sets in the next steps such that all of them are subsets of $A_{i}$. Consider one of these absorbing sets, $A_{i+1}$, such that $A_{i+1} \subseteq A_{i}$. Consider the subgraph $\left(A_{i+1}, E_{A_{i+1}}^{\prime}\right)$ of the digraph $\left(A_{i+1}, E_{A_{i+1}}\right)$ such that $E_{A_{i+1}}^{\prime}$ is simply the restriction of $E_{A_{i}}^{\prime}$ to the nodes of $A_{i+1}$. It is easy to see that in this subgraph, all nodes have indegree and outdegree equal to 1 . Then, the same analysis performed for $\left(A_{i}, E_{A_{i}}^{\prime}\right)$ can be used with $\left(A_{i+1}, E_{A_{i+1}}^{\prime}\right)$ to prove that the agents of $A_{i+1}$ will form symmetric pairs in subsequent steps with one of her maximal houses of the original absorbing set.

\footnotetext{
${ }^{14}$ Notice that, although all nodes of $A_{i}$ form symmetric pairs at step 2, they do not necessarily belong to an absorbing set at this step. Therefore, it may occur that they do not leave the algorithm at the same time. See for instance steps 1 and 2 of Example 2.
} 
By repeating this process for all absorbing sets, we can deduce that the TTAS algorithm assigns, in each absorbing set of the partition determined by Quint and Wako (2004), a sub-allocation such that each agent has one of her maximal houses in this set. Then, applying the result of Quint and Wako (2004), we have that the TTAS algorithm always selects an allocation contained in the strict core when this set is non-empty.

\section{References}

[1] Abdulkadiroğlu, A. and Sönmez, T., (1999). House Allocation with Existing Tenants. J. Econ. Theory. 88, 233-260.

[2] Abdulkadiroğlu, A. and Sönmez, T., (2003). School Choice: A Mechanism Design Approach. Amer. Econ. Rev. 93, 729-747.

[3] Bird, C., (1984). Group Incentive Compatibility in a Market with Indivisible Goods. Econ. Letters. 14, 309-313.

[4] Ehlers, L., (2002). Coalitional Strategy-Proof House Allocation. J. Econ. Theory. 105, 298-317.

[5] Gale, D. and Shapley, L., (1962). College Admissions and the Stability of Marriage. Amer. Math. Monthly. 69, 9-15.

[6] Erdil, A. and Ergin, H., (2008). What's the Matter with Tie-Breaking? Improving Efficiency in School Choice. Amer. Econ. Review. 98, 669-689.

[7] Jaramillo, P. and Manjunath, V., (2009). The difference indifference makes in strategy-proof allocation of objects. Mimeo.

[8] Konishi, H., Quint, T. and Wako, J., (2001). On the Shapley-Scarf Economy: the Case of Multiple Types of Indivisible Goods. J. Math. Econ. 35, 1-15.

[9] Kalai, E. and Schmeidler, D., (1977). An Admissible Set Occurring in Various Bargaining Situations. J. Econ. Theory. 14, 402-411.

[10] Ma, J., (1994). Strategy-Proofness and the Strict Core in a Market with Indivisibilities. Int. J. Game Theory. 23, 75-83.

[11] Quint, T. and Wako, J., (2004). On Houseswapping, the Strict Core, Segmentation, and Linear Programming. Math. Oper. Research 29, 861-877.

[12] Roth, A. E., (1982). Incentive Compatibility in a Market with Indivisibilities. Econ. Letters. 9, 127-132.

[13] Roth, A. E. and Postlewaite, A., (1977). Weak versus Strong Domination in a Market with Indivisible Goods. J. Math. Econ. 4, 131-137.

[14] Roth, Alvin E., Sönmez, T. and Ünver, M.U., (2004). Kidney Exchange. Quart. J. Econ. 119, 457-488.

[15] Shapley, L. and Scarf H., (1974). On Cores and Indivisibility. J. Math. Econ. 1, 23-28.

[16] Sönmez, T. and Ünver, M. U., (2009). Matching, Allocation, and Exchange of Discrete Resources. Handbook of social economics. Elsevier. 
[17] Tarjan, R., (1972). Depth-First Search and Linear Graph Algorithms. SIAM Journal on Computing 2, 146-160.

[18] Yılmaz, Ö., (2009). Random Assignment under Weak Preferences. Games Econ. Behav. 65, 546-558. 